\title{
CATEGORIZACION DE RIESGO CARDIOVASCULAR EN EMPLEADOS DE UNIVERSIDAD NACIONAL AUTONOMA DE HONDURAS, VALLE DE SULA.
}

\author{
Categorization of cardiovascular risk in employees of \\ Universidad Nacional Autónoma de Honduras - Valle de Sula.
}

*Rosa Elena Zúniga, *José Raúl Arita Chávez, *Patricia Monserrath Elvir, *Lesly Altenida Ochoa,
*Ledezna Lizeth Arita, *Virginia Odili Rostran, **Ana Lourdes Girón, **Obed Jeovany Quiroz.

\section{RESUMEN}

Las enfermedades cardiovasculares (ECV) engloban afectaciones tanto del corazón como del cerebro, siendo el problema subyacente la ateroesclerosis como responsable de las enfermedad coronaria y eventos cerebrovasculares, las ECV constituyen la principal causa de morbi-mortalidad a nivel mundial, en su origen se involucran diversos factores, que van desde el estilo de vida y otros que pueden ser modificables como también aquellos que no pueden modificarse como la edad, herencia, entre otros. Objetivo: Identificar riesgo cardiovascular (RCV) mediante Índice de Masa Corporal (IMC)/ índice cintura-cadera y tablas de Framinghan en empleados de la Universidad Nacional Autónoma de Honduras-Valle de Sula, (UNAH-VS) de febrero a octubre, 2016. Pacientes y Métodos: Se realizó investigación cuantitativa, alcance descriptivo y diseño no experimental. La población 210 empleados permanentes, muestra 137 empleados. Muestreo probabilístico estratificado según actividad desempeñada en la Institución. La evaluación fue mediante Instrumento creado por OMS: STEP 3; se midió riesgo cardiovascular por Tabla IMC/índice cintura-cadera y Tablas de Framingham. Los datos se analizaron mediante paquete estadístico SPSS versión 22. Resultados: La categorización de RCV según IMC/índice cintura-cadera fue: muy leve $11(8.05 \%)$, leve $27(19.71 \%)$, moderado $49(35.76 \%)$, alto $26(18.97 \%)$ y muy alto $24(17.51 \%)$. Según Tablas de Framingham: el riesgo de presentar un evento cardiovascular en los próximos 10

\footnotetext{
*Docentes, Escuela Universitaria de las Ciencias de la Salud, Universidad Nacional Autónoma de Honduras, Valle de Sula. EUCS, UNAH-VS.

** Estudiantes de Servicio Social (Carrera de Ciencias de Enfermería y Medicina, respectivamente). EUCS, UNAH-VS.

Dirigir correspondencia a: re_zuniga@hotmail.com

Recibido: 24 de mayo 2017

Aprobado: 30 de Octubre 2017
}

años fue: 131 (95.62\%) bajo, 5 (3.65\%) moderado, y 1 (0.73\%) alto. Conclusión: El riesgo cardiovascular en empleados de UNAH-VS es alto según la categorización $\mathrm{IMC} /$ índice cintura-cadera, la cual estimula a tomar medidas preventivas más tempranamente, en cambio, según Framinghan el riesgo cardiovascular fue menor, esto debido a que incorpora condiciones como diabetes, hipertensión, fumar, niveles de colesterol en sangre.

\section{PALABRAS CLAVE}

Enfermedades cardiovasculares, Factores de riesgo, Prevalencia.

\section{ABSTRACT}

Cardiovascular diseases are one of the main causes of morbidity and mortality worldwide. The factors for this risk can be: non-modifiable and modifiable, based on these factors can be stratified cardiovascular risk. Objective: To identify cardiovascular risk (CVR) using Body Mass Index (BMI) / waist/hip index and Framinghan tables in employees of the National Autonomous University of Honduras-Valle de Sula, (UNAH-VS) from february to october, 2016 Patients and Methods: We performed quantitative research, descriptive scope and non- experimental design. The population 210 permanent employees, sample 137 employees. Sampling probabilistic stratified according to activity performed in the Institution. The evaluation was through an Instrument created by WHO: STEP 3; cardiovascular risk was measured by BMI / waist/hip ratio and Framingham Tables. Results: The categorization of VCR according to BMI / waist-to-hip ratio was very mild $11(8.05 \%)$, mild $27(19.71 \%)$, moderate $49(35.76 \%)$, high $26(18.97 \%)$ and very high 24 (17.51\%). According to Framingham Tables: the risk ofpresenting a cardio- 
vascular event in the next 10 years was: 131 $(95.62 \%)$ low, 5 (3.65\%) moderate, and 1 $(0.73 \%)$ high. Conclusion: The cardiovascular risk in UNAH-VS employees is high according to the BMI/waist-to-hip ratio, which encourages them to take preventive measures earlier, whereas, according to Framinghan, cardiovascular risk was lower, such as diabetes, hypertension, smoking, blood cholesterol levels.

\section{KEYWORDS}

Cardiovascular diseases, Risk factors, Prevalence.

\section{INTRODUCCION}

Las enfermedades cardiovasculares (ECV) son la principal causa de muerte en todo el mundo. Cada año mueren más personas por ECV que por cualquier otra causa. Se calcula que en 2012 murieron por esta causa 17,5 millones de personas, lo cual representa un $31 \%$ de todas las muertes registradas en el mundo. De estas muertes, 7,4 millones se debieron a cardiopatía coronaria, y 6,7 millones a los accidentes cerebro vasculares (AVC). ${ }^{(1)}$

La ECV suelen tener su causa en la presencia de una combinación de factores de riesgo, ${ }^{(1)}$ estos pueden ser no modificables y modificables. Dentro de los factores de riesgo no modificables están la edad, sexo, herencia y antecedentes de eventos cardiovasculares familiares, ${ }^{(2)}$ y dentro de los factores de riesgos personales-modificables están el tabaquismo, hipertensión arterial, sobrepeso corporal, perímetro abdominal, hiperlipidemias por aumento de colesterol total, disminución de colesterol de alta densidad (HDL), aumento del colesterol de baja densidad (LDL) y aumento de triglicéridos, e hiperglucemia. ${ }^{(2)}$ estos son factores de riesgo sobre los que sí se puede actuar para corregirlos, y con ello reducir el riesgo.

El origen multifactorial de las enfermedades cardiovasculares ha permitido que se desarrollen diferentes modelos para evaluar el riesgo cardiovascular, los cuales nos permi- ten prever si nuestra población en estudio desarrollará un evento cardiovascular mortal; el índice cintura/cadera (ICC) es uno de los mejores indicadores antropométricos para predecir el riesgo de enfermedad cardiovascular;,(3) un aumento en el Índice de Masa Corporal (IMC) e Índice cintura/cadera aumenta el riesgo cardiovascular y a la vez el riesgo de padecer diabetes tipo II.(4) La obesidad abdominal por sí sola, es un factor de riesgo cardiovascular en adultos aparentemente sanos. Las diferentes sociedades clasifican el riesgo cardiovascular según la distribución de la adiposidad(4) siendo la distribución central (adbominal) la que conlleva mayor predisposición; actualmente se toma en consideración, que los valores de índice cintura/cadera a partir de los cuales se observa un aumento del riesgo cardiovascular son $>1.0$ en los varones $y>0,85$ en las mujeres. ${ }^{(5)}$

Otra manera de categorizar el RCV es mediante las tablas de Framingham que nos permite predecir el riesgo de sufrir un evento cardiovascular en los próximos 10 años. En 1948, el Servicio de Salud Pública de Estados Unidos inició el Framingham Heart Study, con la finalidad de estudiar la epidemiología y los factores de riesgo de la Enfermedad Cardiovascular. ${ }^{(6)}$ El 11 de octubre de 2013, el Framingham Heart Study celebró 65 años desde el examen de su primer participante en 1948. ${ }^{(7)}$ En los años siguientes, el estudio de Framingham y otros estudios epidemiológicos contribuyeron a identificar los factores de riesgo de enfermedad cardiovascular que ahora se consideran ya clásicos. Framingham creó las tablas que llevan su nombre, donde relaciona tanto los factores modificables como los no-modificables para medir riesgo cardiovascular que varía desde "bajo" hasta "muy alto". ${ }^{(8)}$

El objetivo de esta investigación fue identificar riesgo cardiovascular (RCV) mediante índice de Masa Corporal (IMC) / índice cintura/cadera y tablas de Framinghan en empleados de la Universidad Nacional Autónoma de Honduras-Valle de Sula, (UNAH-VS) de febrero a octubre, 2016. 


\section{PACIENTES Y METODOS}

Se realizó investigación cuantitativa, alcance descriptivo y diseño no experimental, entre febrero a octubre 2016, en empleados permanentes de la UNAH-VS. La población estuvo constituida por 210 empleados permanentes de los departamentos de mantenimiento (45), administración (45) y docentes (122). Se calculó muestreo probabilístico estratificado: 137 empleados, obteniendo: según departamento: mantenimiento (29), administración (29) y docentes (79). Dentro de cada departamento, la elección fue sistemática.

Criterios Incluyentes: Ser empleado permanente de UNAH-VS, ser elegido dentro de la selección por muestreo sistemático dar su consentimiento en participar en la investigación.

Criterio excluyente: No ser empleado de UNAH-VS, no ser empleado permanente, no quedar elegido dentro de la selección por muestreo sistemático, no dar su consentimiento en participar en la investigación, estar embarazada o ser puérpera al momento de la entrevista, ser miembro del equipo investigador.

Previamente, se realizaron cuatro jornadas de inducción a los participantes, explicándoles el objetivo de la investigación y se les solicitó acudir en ayuno el siguiente día, para realizar:

1. Aplicación de instrumento STEPS 3 para la vigilancia de factores de riesgo de enfermedades no infecciosas, creado por la $\mathrm{OMS}^{(9)}$ para registrar información demográfica y relacionada: edad, genero, consumo de tabaco, consumo de alcohol, dieta, actividad física, antecedentes de tensión arterial elevada, antecedentes de diabetes, historia familiar de enfermedad cardiovascular primaria. Además, se creó una ficha de control de datos para cada uno de los empleados seleccionados a los que aceptaron participar en el estudio.

2. Tomar medidas antropométricas, talla, peso, para determinar IMC (Índice de Masa Corporal) y circunferencia de cintura y circunferencia de cadera para determinar el índice cintura/cadera.
Las medidas antropométricas se obtuvieron de la siguiente manera: el peso en balanza robusta y calibrada con precisión de 100 gramos en la pesada, paciente de pie y descalzo. Se tomó altura o talla utilizando un estadiómetro con una calibración de $1 \mathrm{~mm}$ de precisión hasta obtener un plano de Frankfurt correcto.

Se calculó el índice de masa corporal (IMC o índice de Quetelec), resultado de dividir el peso en kg entre la talla en m2. Se considera pre-obesidad un IMC de 25 a 29, obesidad Clase I de 30-34.9, Clase II de 35-39.9, Clase III mayor o igual a 40, Clase IV mayor o igual a 50. ${ }^{(10)}$ Las medidas de la circunferencia de cintura se tomó con cinta métrica inextensible; esta medida se tomó debajo de las costillas flotantes de la caja torácica.

La medida de circunferencia de cadera fue obtenida sobre la máxima curvatura del glúteo, el empleado debió estar de pie y con las piernas juntas, en todas estas medidas se aseguró que el empleado se sintiera cómodo y relajado, ambas medidas se dividen para calcular índice cintura/cadera, en hombre el rango mayor que 1 , y en mujer el rango mayor que 0.85 , son delimitadores del riesgo cardiovascular. ${ }^{(5)}$ Además, se realizó toma de presión arterial con un esfigmomanómetro calibrado.

3. Toma de muestras de sangre en ayuno (entre 10 y 12 horas) al empleado para determinaciones bioquímicas: colesterol total y fraccionado (HDL, LDL, VLDL) triglicéridos, glicemia. Los análisis bioquímicos se realizaron en el Hospital Mario Catarino Rivas.

4. Laboratorio Bussie, financió reactivos para realizar las pruebas bioquímicas: glicemia, colesterol total, colesterol HDL, triglicéridos. El Hospital Mario Catarino Rivas, facilitó equipo de laboratorio: centrifuga, rotador, espectrofotómetro.

EI RCV se caracterizó de la siguiente manera: según el IMC/ índice cintura/cadera, (Ver Tabla No. 1) y Tablas de Medidas de Riesgo cardiovascular según Framingham. 
Tabla No. 1: Valores de riesgo según la distribución de la grasa corporal..

\begin{tabular}{|c|c|c|c|c|c|}
\hline \multirow[b]{2}{*}{ GRADO } & \multirow[b]{2}{*}{ IMC } & Varones & $<0.85$ & $0.85-1.0$ & $>1.0$ \\
\hline & & Mujeres & $<0.70$ & $0.70-0.85$ & $>0.85$ \\
\hline 0 & $20.0-24.9$ & & Muy Leve & Leve & Moderado \\
\hline 1 & $25.0-29.9$ & & Leve & Moderado & Alto \\
\hline 2 & $30.0-34.9$ & & Moderado & Alto & Muy Alto \\
\hline 3 & $35.0-39.9$ & & Alto & Muy Alto & Muy Alto \\
\hline 4 & $\geq 40.0$ & & Muy Alto & Muy Alto & Muy Alto \\
\hline
\end{tabular}

Fuente: Mataix Verdu J, García Diz D, Llopis González D, Técnicas de evaluación del estado nutricional, antropometría y composición corporal. FUNIBER 2016; pag. 106.(11)

Se categorizó RCV utilizando las "Tablas de Framingham" que distribuye los factores de riesgo según sexo, edad, diabetes o no, fuma o no, presión arterial, niveles serológicos de colesterol, y niveles de colesterol HDL. En las tablas se interpola estos factores y los categoriza desde el numero 1 al 29 , si el valor de Colesterol HDL es menor que $35 \mathrm{mg} / \mathrm{dl}$ se debe multiplicar el valor por 1.5 , una vez categorizado, clasifica el riesgo en 4 niveles de la siguiente manera: del número 1 al 5 es riesgo bajo, del 5 al 9 es riesgo moderado, del número 10 al 14 es riesgo alto y mayor que 15 es riesgo muy alto de presentar enfermedad cardiovascular en los próximos 10 años. ${ }^{(12)}$

Se utilizó paquete estadístico SPSS versión 22 y se realizaron análisis descriptivos y de frecuencia. A cada uno de los participantes se les entregó resultados bioquímicos sanguíneos: glicemia, colesterol total, HDL, triglicéridos, además se les explicó su IMC, relación cintura/cadera, con su respectiva categorización de riesgo cardiovascular según IMC/cintura-cadera y Tablas de Framingham. También se les presentó aplicación en línea llamada "Plato inteligente," que les permitiría seleccionar diferentes menús, según sus gustos y preferencias alimenti- cias, haciendo énfasis en el tamaño de las raciones y tipo de alimentos. Y se les motivó a realizar ejercicios por lo menos 45 minutos diarios durante 3 veces en la semana.

Aspectos Éticos: Se presentó protocolo de investigación al Comité de Ética de UNAH-VS, el cual fue aprobado.

\section{RESULTADOS}

Datos Sociodemográficos y Factores de riesgo:

Edad: $16(11.7 \%)$ se encontraban en la edad de 20-34 años, 46 (33.5\%) entre 35-44 años, $36(26.3 \%)$ entre $45-54$ años, $27(19.7 \%)$ entre 55-64 años, 12 (8.8\%) entre 65-74 años. En cuanto al sexo 83 (61\%) eran mujeres y $54(39 \%)$ eran hombres. La población en estudio con antecedentes familiares de enfermedad cardiovascular fueron 63 (46\%) y $74(54 \%)$ sin antecedentes familiares de enfermedad cardiovascular.

De los factores de riesgo cardiovascular modificables, se encontró; sobrepeso y obesidad $77.4 \%$, niveles de colesterol HDL menor que $60 \mathrm{mg} / \mathrm{dl} 76 \%$, sedentarismo $(63 \%)$, bajo consumo de frutas y vegetales $44 \%$ y $30 \%$ respectivamente (Ver Tabla No. 2). 
Resultados de RCV según IMC/índice cintura-cadera:

El $72.27 \%$ (99) de la población en estudio se encontró en riesgo cardiovascular de moderado a muy alto según relación IMC/cintura/ cadera. (Ver Tabla No. 3). Los empleados con abesidad clase II (13), un $76.92 \%$ (10) tienen riesgo cardiovascular muy alto (RCV-MA) y los empleados con obesidad clase III (7), un $85.71 \%$ (6) tenian riesgo muy alto (RCV-MA).

El Riesgo cardiovascular IMC/cintura/cadera relacionado con el sexo se distribuyó de la siguiente manera: de los 83 empleados del sexo femenino; 6 (7.2\%) muy leve, 22 $(26.5 \%)$ leve, $27(32.5 \%)$ moderado, 14 (16.9\%) alto, $14(16.9 \%)$ muy alto. De los 54 empleados del sexo masculino, $5(9.3 \%)$ muy leve, $5(9.3 \%)$ leve, $22(40.7 \%)$ moderado, 12 $(22.2 \%)$ alto, $10(18.5 \%)$ muy alto.
Tabla No. 2: Prevalencia de factores de riesgo cardiovascular modificables.

\begin{tabular}{|l|c|c|}
\hline $\begin{array}{l}\text { Factores Cardiovas- } \\
\text { culares Modificables }\end{array}$ & $\begin{array}{l}\text { No. Em- } \\
\text { pleados }\end{array}$ & $\begin{array}{c}\text { Porcen- } \\
\text { taje }\end{array}$ \\
\hline Fumar & 10 & $7.30 \%$ \\
\hline $\begin{array}{l}\text { IMC mayor que 24.9 } \\
\text { (sobrepeso-obesidad) }\end{array}$ & 106 & $77.40 \%$ \\
\hline Hipertensión & 23 & $16.80 \%$ \\
\hline Diabetes & 9 & $6.70 \%$ \\
\hline $\begin{array}{l}\text { Triglicéridos mayor } \\
\text { que 150 Mg/dl }\end{array}$ & 21 & $15 \%$ \\
\hline $\begin{array}{l}\text { Colesterol mayor } \\
\text { que 200 Mg/dl }\end{array}$ & 6 & $4.40 \%$ \\
\hline $\begin{array}{l}\text { Colesterol HDL menor } \\
\text { que 45 mg/dl }\end{array}$ & 104 & $76 \%$ \\
\hline Sedentarismo & 86 & $63 \%$ \\
\hline Bajo consumo de frutas & 60 & $44 \%$ \\
\hline $\begin{array}{l}\text { Bajo consumo de } \\
\text { verduras }\end{array}$ & 41 & $30 \%$ \\
\hline
\end{tabular}

Fuente: Datos obtenidos después de la aplicación de Instrumento STEP 3 a la población en estudio

Tabla No. 3: Riesgo Cardiovascular según relación IMC e índice cintura/cadera.

\begin{tabular}{|c|c|c|c|c|c|c|}
\hline \multirow{2}{*}{ IMC } & \multicolumn{5}{|c|}{ Riesgo Cardiovascular Cintura/Cadera } & Total \\
\cline { 2 - 7 } & Muy leve & Leve & Moderado & $\begin{array}{c}\text { Alto } \\
\text { H: }>\mathbf{1 . 0} \\
\text { M:>0.85 }\end{array}$ & $\begin{array}{c}\text { Muy alto } \\
\text { H: }>1.0 \\
\text { M:>0.85 }\end{array}$ & \\
\hline $\begin{array}{c}<185 \\
\text { Bajo peso }\end{array}$ & 1 & & & & & 1 \\
\hline $\begin{array}{c}18.5-24.9 \\
\text { Normopeso }\end{array}$ & 10 & 18 & 2 & & & 30 \\
\hline $\begin{array}{c}25-29.9 \\
\text { Pre Obesidad }\end{array}$ & 0 & 9 & 37 & 6 & & 52 \\
\hline $\begin{array}{c}30-34.9 \\
\text { Obesidad Clase I }\end{array}$ & 0 & 0 & 9 & 17 & 8 & 34 \\
\hline $\begin{array}{c}35-39.9 \\
\text { Obesidad Clase II }\end{array}$ & & & 1 & 2 & 10 & 13 \\
\hline $\begin{array}{c}\geq 40 \\
\text { Obesidad Clase III }\end{array}$ & 11 & 27 & 49 & 26 & 24 & 137 \\
\hline Total & & & 1 & 6 & 7 \\
\hline
\end{tabular}

Fuente: Datos obtenidos después de la aplicación de Instrumento STEP 3 a la población en estudio 
Relación de factores de riesgo cardiovascular e índice cintura/cadera:

Se presenta a continuación porcentajes de empleados que presentaron hipertensión arterial, diabetes, sedentarismo, fumaban y que presentaron índice cintura/cadera arriba de parámetros aceptados como valores delimitadores del riesgo cardiovascular.

Los empleados que presentaron hipertensión arterial fueron: 12 hombres, $11(91.6 \%)$ de ellos presentaron un índice cintura/cadera mayor que 1.0. En cuanto a las mujeres 11 presentaron hipertensión arterial y 7 (63.63\%) de ellas tenían un índice c/c mayor que 0.85 .

Los empleados que presentaron diabetes fueron: 7 hombres, 6 (85.7\%) de ellos presentaron índice cintura/cadera mayor que $1.0 \mathrm{y}$ las mujeres fueron 2 , y $1(50 \%)$ con índice cintura/cadera mayor que 0.85 . En cuanto a los fumadores eran 10 y el $80 \%$ (8) presentó índice cintura/cadera mayor que 1.0 ó 0.85 según fuese hombre o mujer.

De los empleados que presentaron sedentarismo (86) y que presentaban también índice cintura/cadera mayor que 1.0 y 0.85 según fuese hombre ó mujer, el $72.09 \%$ (62) presentaron esta condición, el resto 27.91 (24) solamente presentaron sedentarismo.

Resultados de Riesgo Cardiovascular según Tablas de Framingham

Según Tablas de Framingham: 128 (93.43\%) tenían RCV bajo. (Ver Gráfica No.1).

Gráfico No. 1: Categorización de Riesgo Cardiovascular según Tablas de Framingham.

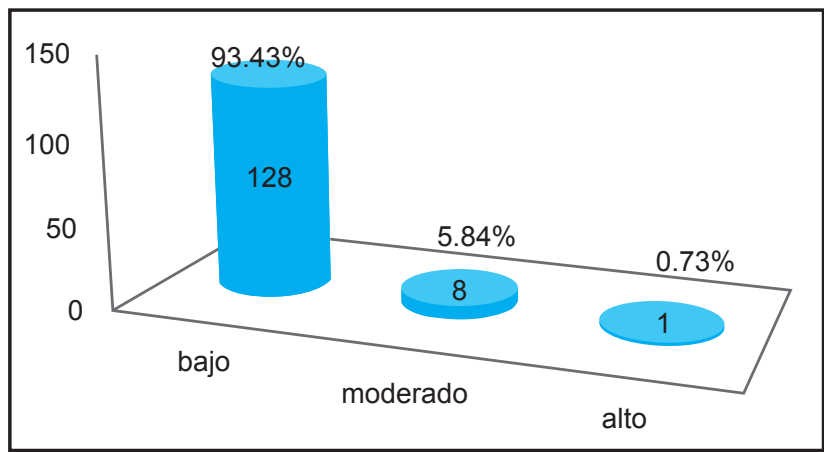

Fuente: Datos obtenidos después de la aplicación de Instrumento STEP 3 a la población en estudio

\section{DISCUSION}

La predicción del riesgo cardiovascular proporciona una herramienta útil para establecer prioridades en la atención primaria, con el objetivo de acercarnos más a la realidad multifactorial de las enfermedades cardiovasculares y a su prevención. ${ }^{(13)}$

En estudios realizados utilizando una predicción y categorización de riesgo cardiovascular donde se mide grasa abdominal mediante el índice cintura/ cadera e IMC, se observó que existe una relación bien cercana entre los pacientes con índices cintura-cadera arriba de 1.0 o 0.85 con enfermedades (diabetes, hipertensión arterial) y hábitos (sedentarismo, fumar). Así lo demuestra investigación hecha en Argentina (Ciudad de Corrientes) donde el $91.37 \%$ de la población en estudio que presentaron un índice cintura/cadera mayor que 1.0 o 0.85 según fuese hombre o mujer presentaban enfermedades predisponentes como ser diabetes o hipertensión. ${ }^{(14)}$

En la presente investigación, las personas que presentaron hipertensión arterial, diabetes, sedentarismo, consumo de tabaco en su gran mayoría tuvieron índice cintura/cadera arriba de 1.0 o 0.85. Se podría afirmar la utilidad de esta medición como predictor de evento cardiovascular futuro. ${ }^{(14)}$

En la categorización según las tablas de Framingham se prioriza la presencia de los siguientes factores de RCV: diabetes, hipertensión arterial, consumo de tabaco, hiperlipidemias.

También se han realizado investigaciones para categorizar RCV con Tablas de Framingham en Universidades como: Universidad de Chihuahua, México, donde se presentó un $32.2 \%$ de "riesgo alto" en hombres, atribuible a que presentaron dislipidemias el $50 \%$ de los hombres que participaron en la investigación $^{(15)}$, diferente a nuestra investigación que el "riesgo alto" fue de $1.85 \%$ (1), aunque también con presencia de dislipidemia, sin embargo en investigación realizada en Ecuador en la ciudad de Sucúa - Morona Santiago, los resultados fueron: el riesgo cardiovascu- 
lar bajo se presentó en la mayoría de la muestra $(96,8 \%)$, seguido por el riesgo cardiovascular moderado con un $2,8 \%$, y finalmente un $0,4 \%$ de presencia de riesgo cardiovascular alto (8), todos estos porcentajes similares al de la presente investigación.

En revisión bibliográfica de publicaciones de países de Latinoamérica, durante el período 2010-2015, que fueron seleccionadas en inglés y español de bases de datos de PubMed, OMIM, Scielo, EBSCO y revistas de cardiología, se concluyó que los latinoamericanos tienen una alta prevalencia de sobrepeso/obesidad, sedentarismo, tabaquismo como factores de riesgo cardiovascular sin diferencias significativas por sexo, ${ }^{(2)}$ al igual que en la presente investigación el sobrepeso/obesidad y sedentarismo fueron los factores de RCV con mayor prevalencia.

La obesidad por sí sola es un factor de riesgo para la salud de la población ya que determina el desarrollo y progresión de diversas patologías enmarcadas dentro de la definición de enfermedades cardiovasculares (ECV), tales como la hipertensión arterial, las dislipidemias, la arterioesclerosis, la diabetes. ${ }^{(16)}$

Datos actualizados refieren que en Honduras el $51 \%$ de las mujeres sufren de sobrepeso y obesidad y que es el grupo de 15 a 19 años de edad el menos afectado (21\%), pero hay un incremento al doble en el grupo de 20 a 29 años de edad $(44,8 \%) .{ }^{17)}$ En la presente investigación; $60(72.3 \%)$ de las mujeres participantes presentaron sobrepeso/obesidad con la siguiente distribución: 32 (53.3\%) con sobrepeso, 15 (25\%) con Obesidad Clase I, 8 (13.3\%) Obesidad Clase II, 5 (8.3\%) Obesidad Clase III, siendo estos valores más altos que los que refieren los valores para Honduras como país en cuanto a sobrepeso/obesidad, y la edad con mayor predominio en esta condición es de 35-44 años.

En Tegucigalpa, capital de Honduras, se estima que el $5,4 \%$ de la población está afectada por diabetes tipo II, de los cuales el $50 \%$ tiene diagnóstico previo y la prevalencia aumenta a $7,9 \%$ en personas mayores de
40 años de edad. Dos de cada cinco personas tienen antecedentes de hipertensión arterial; una de cada cinco personas está obesa, y el $51,7 \%$ tiene sobrepeso y obesidad. También se identifica que el hábito de fumar afecta al 7,3\% de los participantes. ${ }^{(18)}$

Nuestra población en estudio presentó una prevalencia en diabetes $(6.7 \%)$ casi similar al de la población en Tegucigalpa, hipertensión arterial en menor prevalencia, obesidad en mayor prevalencia y el hábito de fumar en igual prevalencia.

Otro punto interesante a resaltar es que no hubo mucha diferencia en cuanto a riesgo cardiovascular entre los que tenían antecedentes familiares de enfermedad cardiovasculares y los que no tenían éstos antecedentes familiares.

En Honduras hay algunos estudios sobre factores de riesgo cardiovascular pero no se encontró ninguno que categorizara este riesgo ya sea con $\mathrm{IMC/}$ cintura/cadera o por medio de las Tablas de Framingham.

Es importante señalar que muchos individuos en la población general tienen uno o más factores de riesgo de ECV y en estudios realizados en diversos países han demostrado que más del $90 \%$ de los eventos ocurren en individuos con al menos un factor de riesgo. ${ }^{(19)}$

En conclusión, el riesgo cardiovascular en empleados de UNAH-VS es alto según la categorización IMC/índice cintura/cadera. Estas personas aparentemente sanas deben tener una intervención en su estilo de vida para tener cambios radicales. En cuanto a la categorización de riesgo cardiovascular según Framinghan el riesgo fue bajo.

Se Recomienda, elaborar programas dentro de la UNAH, para promover estilos de vida saludables, particularmente con dietas alimenticias con un valor calórico y nutricional adecuada, ejercicio constante y así de esta manera prevenir enfermedades no contagiosas como las enfermedades cardiovasculares y metabólicas. 


\section{REFERENCIAS BIBLIOGRÁFICAS}

1. Organizacion Mundial de la Salud. Enfermedades cardiovasculares [Internet]. Ginebra: OMS; 2015 [Actualizada 2017, citado el 5 de enero 2016].

Disponible en: http://www.who.int/media centre/factsheets/fs317/es/.

2. Pereira-Rodríguez J, Peñaranda-Florez D, Reyes-Saenz A, Caceres-Arevalo K, Cañizarez-Pérez Y. Prevalencia de factores de riesgo cardiovascular en América Latina: una revisión de la evidencia publicada de 2010 a 2015. Rev. Mex. Cardiol. [Internet]. 2015 [Citado 10 mayo 2017]; 26(3): 125-139. Disponible en: http:// $w w w . s c i e l o . o r g . m x / s c i e l o . p h p ? s c r i p t=$ sci_arttext\&pid=S0188-21982015000 $300004 \&$ Ing $=$ es.

3. Gharakhanlou R, Farzad B, Agha-Alinejad H, Steffen LM, Bayati M. Anthropometric measures as predictors of cardiovascular disease risk factors in the urban population of Iran. Arq Bras Cardiol 2012; 98(2): 126-35.

4. González-Chávez A, Amancio-Chassin $O$, Islas-Andrade $S$, Revilla-Monsalve $C$, Hernández-Q M, Lara-Esqueda $A$, et al. Factores de riesgo cardiovascular asociados a obesidad abdominal en adultos aparentemente sanos. Rev Med Inst Mex Seguro Soc, 2008; 46(3): 273-79.

5. Consenso SEEDO'2000 para la evaluación del sobrepeso y la obesidad y el establecimiento de criterios de intervención terapéutica. Med Clin. 2000; 115(15): 587-97.

6. Dawber TR, Meadors GF, Moore FE Jr. Epidemiological approaches to heart disease: the framingham study. Am J Public Health Nations Health. 1951; 41(3): 279-81.

7. Mahmood SS, Levy D, Vasan RS, Wang TJ. The framingham heart study and the epidemiology of cardiovascular diseases: a historical perspective. Lancet. 2014 15;383(9921):999-1008.

8. Arboleda Carvajal M, García Yánez A. Riesgo cardiovascular: análisis basado en las tablas de framingham en pacientes asistidos en la unidad ambulatoria 309, IESSS - SUCÚA. Rev. Fac. Med [internet]. 2017 [Citado 4 octubre 2017]; 25(1):20-30. Disponible en: http://www. scielo.org.co/scielo.php?script=sci_art text\&pid=s0121-52562017000100003\&l ng=en. http://dx.doi.org/10.18359/rmed. 1949.

9. Organización Mundial de la Salud. Manual de vigilancia STEPS de la OMS: el método STEPwise de la OMS para la vigilancia de los factores de riesgo de las enfermedades crónicas. Ginebra: OMS; 2006.

10. Salas-Salvadó J, Rubio MA, Barbany M, Moreno B. Consenso SEEDO 2007 para la evaluación del sobrepeso y la obesidad y el establecimiento de criterios de intervención terapéutica. Med Clin. 2007; 128(5):184-96.

11. Mataix Verdú, Francisco José. Nutrición y alimentación humana. $2^{\mathrm{a}}$ ed. Madrid: Ergón; 2015.

12. Generaltat de Catalunya. Tablas para el cálculo del riesgo coronario a 10 años: adaptación de las tablas de Framingham a la población Española. [Internet]. Catalunya: Generaltat. 2012 [Citado el 2 de junio 2016]. Disponible en: www.regicor. org/media/upload/pdf/taules_2012_cas tella_editora_44_1_1.pdf.

13. Vega Abascal J, Guimará Mosqueda M, Vega Abascal L. Riesgo cardiovascular, una herramienta útil para la prevención de las enfermedades cardiovasculares. Rev Cubana Med Gen Integr. [Internet]. 
2011 [Citado 2 sept 2017]; 27(1): 91-97. Disponible en: http://scielo.sld.cu/scielo. php? script $=$ sci_arttext\&pid=S0864-21 252011000100010\&lng=es.

14. Gavilan VE, Goitia JI, Irala GR, Luzuriaga MG, Rodríguez S, Costa JA, et al. Valoración del índice cintura-cadera y su correlación con el riesgo cardiovascular en un hospital de la Ciudad de Corrientes. Argentina: UNNE. 2002. Disponible en: http://www.unne.edu.ar/unnevieja/ Web/cyt/cyt/2002/03-Medicas/M-057. pdf.

15. Sáenz Carrasco JA, Muñoz Daw MJ, Hinojos Seáñez E, De la Torre Díaz ML, Riesgo cardiovascular en los empleados de la Universidad Autónoma de Chihuahua, México. Nutr Clín Diet Hosp. 2016; 36(3): 45-52.

16. Ascaso J, González Santos P, Hernández Mijares A, Mangas Rojas A, Masana L, Millán J, et al. Management of dyslipidemia in the metabolic síndrome: recommendations of the Spanish HDL Forum. Am J Cardiovasc Drugs. 2007; 7(1): 39-58.
17. Instituto Nacional de Estadísticas. Honduras- Encuesta nacional de demografía y salud 2011-2012. [Internet] Tegucigalpa: INE; 2013. [Actualizada el 2016, citado el 12 de mayo del 2016]

Disponible en: https://dhsprogram.com/ pubs/pdf/GF28/GF28.pdf.

18. Organización Panamericana de la Salud. Iniciativa Centroamericana de Diabetes (CAMDI): encuesta de diabetes, hipertensión y factores de riesgo de enfermedades crónicas, Belice, San José, San Salvador, Ciudad de Guatemala, Managua y Tegucigalpa, 2009. Washington: OPS; 2010.

19. Yusuf S, Hawken S, Ounpuu S, Dans T, Avezum A, Lanas F, et al. Effect of potentially modifiable risk factors associated with myocardial infarction in 52 countries (the INTERHEART study): case-control study. Lancet. [Internet] 2004 Sep 11-17 [Citado el 20 de octubre 2017]; 364(9438): 937-52. Disponible: https://www.ncbi.nlm.nih.gov/pubmed/ 15364185. 